\section{Tumour suppressor genes and risk of metastasis in ovarian cancer}

\section{W S Lowry, R J Atkinson}

Department of Oncology, Queen's University of Belfast, Belfast BT9 7BL W S Lowry, professor RJ Atkinson, senior lecturer

Correspondence to: Professor Lowry.

$B M 7$ 1993;307:542
The silent onset of ovarian cancer often leads to metastatic spread before diagnosis can be made. The cause remains unknown, but molecular studies are beginning to reveal some mechanisms. Inactivation of tumour suppressor genes is thought to be responsible for initiating many forms of cancer. Studies of allele loss indicate areas of genome where inactivation may occur. We recently examined the relation between the clinical stage of epithelial ovarian cancer and allele loss at three loci on chromosome 17: progressive loss was detected with advancing stages of disease. ${ }^{1}$

The clinical stage of a tumour defines the extent of the disease and is the best determinant of prognosis for most forms of cancer. It depends on the time elapsed before diagnosis, hence the value placed on early detection. The histopathological grade, however, better describes the intrinsic nature of the tumour: well differentiated tumours are slow growing whereas undifferentiated, anaplastic tumours metastasise rapidly. Thus poorly differentiated tumours tend to be diagnosed at a more advanced stage. At the molecular level one might anticipate less damage to genetic material with well differentiated tumours.

\section{Methods and results}

We collected 52 fresh specimens of ovarian tumours, of which 38 satisfied the pathological criteria of invasive malignant disease. Complete clinical and laboratory data were available for 33 of the cases, but three were homozygous and thus uninformative. The remaining 30 cases were informative at one or more of the three loci examined on chromosome 17 (two polymorphic loci (YNZ22.2 and BHP53) on 17p13 and one at the THH59 locus (17q23-qer)). DNA was extracted from fresh blood samples and was analysed by Southern blot hybridisation. The tumours were classified histologically into well, moderately, and poorly differentiated lesions according to grades I, II, and III respectively.

The greatest allele loss was seen in grade III tumours at all three loci, with the highest loss of over $90 \%$ at THH59) (table). Allele loss was least in grade I tumours, with no loss at $17 \mathrm{p}$. Results for grade III tumours were compared with combined results for grade I and II lesions at each locus with Fisher's test of exact probability (two tailed): significantly greater allele loss was associated with grade III lesions at THH59 (11/12 $v 2 / 10, \mathrm{p}=0.002)$ and YNZ22 (10/12 $v$
Allele loss at three loci on chromosome 17 by histopathological grade of tumour among 30 cases of ovarian cancer. Values are proportions of those cases that were informative at each of the loci

\begin{tabular}{lccc}
\hline & \multicolumn{3}{c}{ Grade of tumour } \\
\cline { 2 - 4 } $\begin{array}{l}\text { Loci on } \\
\text { chromosome 17 }\end{array}$ & III & II & I \\
\hline THH59 & $11 / 12$ & $1 / 3$ & $1 / 7$ \\
YNZ22 & $10 / 12$ & $1 / 6$ & $0 / 4$ \\
p53 & $3 / 5$ & $1 / 2$ & $0 / 2$ \\
\hline Total & $24 / 29$ & $3 / 11$ & $1 / 13$ \\
\hline
\end{tabular}

$1 / 10, p=0.002$ ), but the difference between the small number of observations for p53 was not significant $(3 / 5 v 1 / 4, \mathrm{p}=0 \cdot 714)$. When the results for the three loci were combined the difference between grade III lesions and grade I and II lesions was highly significant $\left(24 / 29\right.$ v 4/24; $\left.\chi^{2}=20.44, \quad \mathrm{df}=1 ; \mathrm{p}<0.001\right)(95 \%$ confidence interval for the difference $45 \%$ to $87 \%$ ).

\section{Comment}

The histological grade of a tumour is difficult to define and measure accurately, and it may deteriorate with advancing malignancy as cells undergo multiple abnormal divisions. Despite such criticisms, however, most clinical and pathological studies agree that grading is related to survival. ${ }^{2}$ In our previous analysis of tumour stages, allele loss on $17 \mathrm{p}$ was seen as a late step in the progression of ovarian malignancy. This seems to be ubiquitous in many tumours and is probably related to inactivation of the p53 tumour suppressor gene. Allele loss at THH59 is an earlier step and appears to be specific to ovarian cancer.

Prognostic factors can be analysed more precisely when staging and grading are considered together. Evidence suggests that loss of a putative tumour suppressor gene at THH59 is significantly higher in undifferentiated cancers. These rapidly growing tumours have a greater potential for metastatic spread even in early stage disease. The highly significant difference in the cumulative observations strongly suggest that anaplastic tumours have lost the entire chromosome. The degree of malignancy of the tumour thus depends on the extent of underlying molecular damage.

We thank our colleagues in gynaecology and pathology; $\mathrm{Dr}$ Hilary Russell, who initiated the earlier laboratory studies; and Mrs Patricia White for technical assistance.

1 Eccles DM, Russell SEH, Haites NE, Atkinson R, Bell DW, Gruber L, et al. Early loss of heterozygosity on $17 \mathrm{q}$ in ovarian cancer. Oncogene 1992;7: 2069-72.

Anderson MC. In: Sharp F, Soutter WP, eds. Ovarian cancers-the way ahead Section 2. Chichester: Wiley, 1987:63-4.

(Accepted 10 May 1993)

\section{MRCGP pass rate by medical school and region of postgraduate training}

\author{
Richard Wakeford, John Foulkes, \\ Chris McManus, Lesley Southgate
}

Initiatives to measure and enhance the quality of higher education for both undergraduates and postgraduates (academic audit, teaching quality assessment) will shortly start to impact on medical schools in the United Kingdom.

Mr Wakeford.

$B M \mp 1993 ; 307: 542-3$
We believe that it would be helpful to consumers of medical education (prospective students), to its providers (medical schools and their curriculum designers, postgraduate training schemes), and to its customers (medical care providers) if comparative output data on medical schools and postgraduate training were available. Without a national medical qualifying examination this is probably possible only by examining the performance of candidates for the major postgraduate examinations of the royal colleges.

\section{Methods and results}

To start such a process we analysed the pass-fail result of seven recent diets (December 1988-December 1991) of the membership examination of the Royal College of General Practitioners (MRCGP) by clinical 